\title{
Ludwig Schläfli - ein genialer Schweizer Mathematiker
}

\author{
Ruth Kellerhals
}

\section{Einleitung}

Aus Anlass des 100-jährigen Bestehens der Schweizerischen Mathematischen Gesellschaft soll an Ludwig Schläfli erinnert werden, der zu den ganz grossen Schweizer Mathematikern gehört. Es ist mir eine Ehre diese Aufgabe zu übernehmen. Ich stütze mich dabei auf meine Antrittsvorlesung „,Der Mathematiker Ludwig Schläfli (15.01.1814 20.03.1895)“" vom 2. Februar 1996 im Rahmen der Habilitation an der Universität Bonn, die anlässlich des 100. Todestages von Schläfli entstanden ist. Der Vortrag ist in den DMVMitteilungen 1996 veröffentlicht worden (cf. [5]) und wird hier mit deren Einverständnis etwas modifiziert und verkürzt wiedergegeben. Im Folgenden möchte ich vor allem die beeindruckende Persönlichkeit des Wissenschaftlers Ludwig Schläfli hervorheben und dazwischen einen kleinen Einblick in Teile seines Schaffens geben.

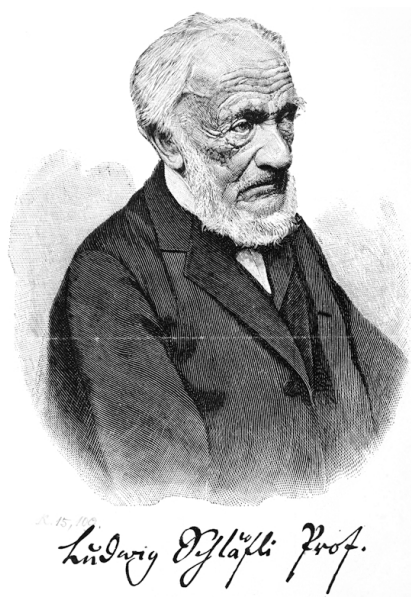

Abb. 1 Stich mit Portrait von Schläfli (Reproduktion aus der Graphischen Sammlung der Schweizerischen Nationalbibliothek in Bern). 
Schläflis gesamter Nachlass wurde von seinem Schüler und späteren Nachfolger J.H. Graf gesammelt und katalogisiert. Nach Grafs Verzeichnis hat das Steiner-Schläfli-Komitée 60 der 65 mathematischen Arbeiten von Schläfli geordnet und in 3 Bänden Gesammelte Mathematische Abhandlungen bei Birkhäuser Basel von 1950 bis 1956 herausgegeben (vgl. [8]). Leider sind die Abhandlungen seit geraumer Zeit vergriffen.

Von Graf stammt auch ein erster und sehr aufschlussreicher Aufsatz [3] über das Lebenswerk seines Lehrers. Einen zweiten [1] hat J.J. Burckhardt 1948 verfasst. Daneben existieren nur wenige Veröffentlichungen über die Person Schläfli. Ich stütze mich neben der am Schluss aufgeführten Literatur auf die Briefwechsel, die Schläfli mit Borchardt, Cayley und Steiner geführt hat. Dies sind

Der Briefwechsel zwischen Jakob Steiner und Ludwig Schläfli

Mitteilungen der Naturforschenden Gesellschaft in Bern (1897), 61-264.

Briefwechsel von Ludwig Schläfli mit Arthur Cayley

Herausgeber J.H. Graf, K.J. Wyss, Bern 1905.

Briefwechsel von Ludwig Schläfli mit C.W. Borchardt (1856-1877)

Mitteilungen der Naturforschenden Gesellschaft in Bern (1915), 50-69.

Dem Artikel [7] von Otto Schlaginhaufen, Professor der Anthropologie, kann man neben vielem makaber Anatomischen entnehmen, dass Schläfli klein gewachsen und nur knapp 1,60 m gross war. Dagegen besass er einen ungewöhnlich, ja pathologisch grossen Hirnschädel (Kapazität $1800 \mathrm{~cm}^{3}$, Gewicht 1566 gr), mit ausgeprägter Stirnregion, was in seinem Reisepass vermerkt worden ist. Zudem fielen grosse Augenhöhlen auf. Nach damaligen Untersuchungen besass er das drittgrösste je gemessene Hirngewicht, und es ist der Reihenfolge nach grösser als jenes von Lejeune Dirichlet, Gauss, Steiner und abgeschlagen - Kant.

\section{Die erste Lebenshälfte}

Bevor ich zu den wissenschaftlichen Leistungen Schläflis komme, möchte ich kurz dessen ersten Lebensabschnitt skizzieren.

Ludwig Schläfli wurde am 15. Januar 1814 in Grasswil (Kanton Bern) geboren, als Sohn des Kaufmanns Johann Ludwig Schläfli und der Magdalena, geborene Aebi. Er wuchs als Ältester von drei Brüdern und einer Schwester in Burgdorf auf.

Seine mathematische Begabung trat schon früh in Erscheinung. So schreibt er im Rückblick Folgendes an seinen Freund Hugo Schiff in Florenz:

\section{Schläfli an H. Schiff}

An eine Autobiographie werde ich nicht Hand legen, denn mein Leben war zu unbedeutend; alle jungen Männer, mit denen ich fort und fort bekannt werde, haben mehr Bewusstsein, als ich im selben Alter gehabt habe. Das Wenige, was ich sagen kann, will ich Ihnen sogleich hin schreiben.

Als ich in die Bürgerschule kam und als unterster (kleinster) auf der hintersten Bank sass, zeichnete der alte Lehrer auf meine Schiefertafel ein Quadrat und einen Kreis und zwar sehr gut und hiess mich das nachmachen und die Tafel damit füllen. Ich hatte daran eine 
unvergessliche Freude. - Die Ausziehung der dritten Wurzel lernte ich von einem Schwager meiner Mutter, der Weber und Trüllmeister, vielleicht auch Feldmesser war. Die Destillation und die Bereitung des Wasserstoffs aus Wasserdampf und glühendem Eisen erklärte mir ein Oheim, der Arzt war, und schloss mir damit eine neue Welt auf. Ein Schulkamerad, der von einem deutschen Schuhmacher (der, wie es scheint, Freude an der Erkenntniss der Natur hatte) ein Buch geliehen hatte, las aus demselben in der Schulstube vor der Ankunft des Lehrers die Kepler'schen Gesetze vor und sprach sich über die allgemeine Gravitation aus. Das wirkte wie ein Blitz in der Finsterniss.

Sein Vater wusste aber lange nicht, was er aus seinem Sohn machen sollte. Zunächst dachte er an eine Art Hausierer: Mit einem Korb voll Waren sollte er die umliegenden Dörfer besuchen, die Preise der Gegenstände wurden ihm eingeschärft. Doch schon nach einer Woche kehrte er abgemagert und hungrig zurück, verkauft hatte er fast nichts, weil er nicht begreifen konnte, dass man eine Ware teurer verkaufe, als man sie eingekauft habe. So liess man denn Ludwig studieren!

Als 15-jähriger erhielt er ein Stipendium, absolvierte das Gymnasium in Bern und studierte anschliessend Theologie an der Universität Bern. Daneben vertiefte er sich aber mehr und mehr in die Mathematik. Bevor er sein Theologiestudium mit dem Staatsexamen 1838 abschloss, trat er, zunächst als Vikar, eine Lehrerstelle für Mathematik und Naturkunde an der Bürgerschule (Progymnasium) in Thun an, was ihm mehr behagte. Zum Pfarramt fühlte er sich nicht berufen. Seinen Eltern erklärte er, ,er könne nicht alles glauben“.

Er unterrichtete 10 Jahre lang in Thun. In seiner wenigen Freizeit widmete er sich mit ganzem Eifer dem Studium der höheren Mathematik: So sass er regelmässig ab 3 Uhr morgens! - vor dem Unterricht am Schreibtisch und arbeitete.

Nebenbei, auch verbunden mit seiner Lehrtätigkeit, interessierte er sich für Botanik und wurde zum anerkannten Experten. Davon zeugen zwei Vorlesungen über geometrische Botanik, welche er in den Jahren 1853/54 an der Universität Bern hielt, sowie wertvolle Aufzeichnungen über die Flora des Kantons Bern (cf. [6]).

Bei dem Autodidakten Schläfli wuchs langsam das Bedürfnis, bei den damaligen Kapazitäten der Mathematik, Steiner, Jacobi und Lejeune Dirichlet, zu lernen und Anregungen zu bekommen.

Als Jakob Steiner, der Professor in Berlin war, zu Besuch in Bern weilte, vermittelte ein gemeinsamer Bekannter ein Zusammentreffen der beiden. Steiner war sehr beeindruckt von Schläfli, seinen mathematisch profunden Kenntnissen und stichhaltigen Beweisführungen und seinem scharfen analytischen Verstand. Und er beschloss, sich Schläflis anzunehmen, dies allerdings auf seine eigene Weise: Steiner bot Schläfli an, ihn, Jacobi und Dirichlet nach Rom zu begleiten und den Winter 1843/44 dort zu verbringen. Da gäbe es genug Möglichkeit zum Fachsimpeln. Seinen Kollegen dagegen erklärte er, dass er in Bern einen ländlichen Mathematiker kenne, für die Welt ein Esel und genialster Tölpel, der ihm je vorgekommen sei, der aber Sprachen lerne wie ein Kinderspiel und den man somit als Dolmetscher mitnehmen könne! Tatsächlich findet man in Schläflis Nachlass Notizen und Hefte mit substantiellen Sprachstudien beispielsweise über Arabisch, Englisch, Französisch, Griechisch, Hebräisch, Italienisch, Koptisch, Neupersisch, Polnisch, Russisch, Sanskrit und Rigveda (über 90 Hefte), Schwedisch und Türkisch. 
Dennoch kann die Reise nach Rom als wichtige Station und Wendepunkt im Leben Schläflis bezeichnet werden. Dort erhielt er täglich morgens Unterricht von Dirichlet in Zahlentheorie, und er übersetzte mathematische Arbeiten von Jacobi und Steiner ins Italienische.

Mit Steiner führte er im Anschluss einen intensiven Briefwechsel, der ihn mit mathematischen Hausaufgaben ständig eindeckte.

\section{Schläfli an J. Steiner}

Ihr letzter Brief vom 31. Juli 1851 hat mich herzlich gefreut; nur muss ich jetzt zu meiner Beschämung gestehen, dass ich an den darin angeregten schweren und schönen Sachen bis jetzt noch nicht gearbeitet habe, dass ich aber nun bald Fleiss darauf verwenden werde, darf ich jetzt um so mehr versprechen, weil ich unlängst (November) einen der für mich seltenen glücklichen Augenblicke im Studium der Mathematik erlebt habe.

Entschuldigen Sie es mit meiner armseligen Lage, dem fast gänzlichen Mangel an geselligem Verkehr und der daraus hervorgehenden gedrückten Stimmung, dass ich fast den ganzen Sommer der morphologischen Botanik widmete.

Wenn ich nur wüsste, die reine Mathematik mit objektiver Wirklichkeit zu verbinden! Es war früher mein Wunsch, mathematische Physik zu studiren; aber wenn man nicht die Mittel hat, um eigene Versuche (zu machen), so ist da kaum etwas zu leisten.

Zurück in Thun verspürte Schläfli zunehmend das Bedürfnis, sich ausschliesslich der Mathematik zu widmen. Er bewarb sich um die venia legendi und eine Anstellung an der Universität Bern. 1848 wurde er zum Privatdozenten ernannt und erhielt ein bescheidenes Dozentenhonorar von nur 400 Franken pro Jahr. Dazu gibt es folgende Anekdote: Die Steuerbehörde des Kantons Bern mochte nicht glauben, dass jemand von lediglich 400 Franken pro Jahr existieren könne, veranlagte ihn zum doppelten Gehalt und brummte ihm erst noch Verzugszins auf. Schläfli wehrte sich nicht. Mit diesem Hungerlohn musste er sich noch bis zu seiner Ernennung zum ausserordentlichen Professor im Jahre 1853 über Wasser halten, eine Beförderung, die nur auf massiven Druck von Steiner erfolgte. Materiell war dies eine schwere Zeit für Schläfli. So schreibt er folgende Zeilen an das Erziehungsdepartement in Bern:

\section{Schläfli an die Erziehungsdirektion in Bern}

Ich bin daher durch meine Habilitation an hiesiger Hochschule, zu welcher ich unter Eröffnung günstiger Aussichten von der Behörde aufgefordert worden, in der That in die drückendste Lage gerathen.

Eigenes Vermögen habe ich nicht; das kleine Erbe, das mir von meinen Eltern selig zugefallen, habe ich seiner Zeit der Waisenbehörde von Burgdorf zur Unterhaltung meiner unglücklichen imbecillen Schwester abgegeben; beschränkt einzig auf das Honorar von 400 Franken, muss ich im eigentlichen Sinne des Wortes darben, nicht nur an meiner Person, was ich mit Freuden ertrüge, sondern auch an allen Hülfsmitteln meiner Wissenschaft.

Seinen Unterhalt musste er sich von 1854 bis 1860 zusätzlich mit Berechnungen für die Schweizerische Nationalversicherungskasse verdienen, was einen grossen Teil seiner Energie in Anspruch nahm. 


\section{Zu Schläflis mathematischen Hauptresultaten}

Es ist unmöglich, einen vollständigen Einblick in Schläflis mathematisches Werk zu vermitteln. Er war sehr vielseitig tätig, und dementsprechend sind die Gesammelten Mathematischen Abhandlungen nicht nach Fachrichtung, sondern - bis auf wenige Ausnahmen - chronologisch angeordnet.

In den Abhandlungen fallen zwei dicke Arbeiten auf, beide stammen aus Schläflis mathematischer Jugendzeit.

\section{Zur Eliminationstheorie und algebraischen Geometrie:}

Über die Resultante eines Systems mehrerer algebraischer Gleichungen: Ein Beitrag zur Theorie der Elimination, 1852 (104 Seiten).

\section{Zur Geometrie in $\boldsymbol{n}$ Dimensionen:}

Die Theorie der vielfachen Kontinuität, 1850-1852 (239 Seiten).

Im Folgenden werde ich nur auf diese beiden fundamentalen Arbeiten eingehen und verweise für Schläflis Beiträge zur Differentialgeometrie, Analysis, Theorie der elliptischen Funktionen, Modulfunktionen, Zahlentheorie, Mechanik und Himmelsmechanik auf [5] und [8].

I. Die Arbeit über die Eliminationstheorie ist von der Akademie der Wissenschaften in Wien veröffentlicht worden. Im zweiten Teil der Arbeit befasst sich Schläfli mit Anwendungen auf allgemeine algebraische Mannigfaltigkeiten und insbesondere auf Flächen dritten Grades

$$
F^{3}=\left\{(x, y, z) \in \mathbb{R}^{3} \mid f(x, y, z)=0\right\},
$$

wobei

$$
f(x, y, z)=\sum_{0 \leq i+j+k \leq 3} a_{i j k} x^{i} y^{j} z^{k}, \quad a_{i j k} \in \mathbb{R},
$$

ein Polynom dritten Grades in den reellen Variablen $x, y, z$ ist. Darauf aufbauend und inspiriert durch den Briefwechsel mit Steiner, der sich intensiv mit Kubiken (etwa der Römerfläche) beschäftigte und ihm auch von Cayleys Entdeckung der 27 Geraden auf einer glatten Kubik berichtete, befasste sich Schläfli eingehend mit Kubiken.

In den zwei wichtigen Arbeiten

- An attempt to determine the 27 lines upon a surface of third order, and to divide such surfaces into species in reference to the reality of the lines upon the surface, 1858,

- On the distribution of surfaces of the third order into species, in reference to the absence or presence of singular points, and the reality of their lines, 1863,

klassifizierte er die Kubiken in Abhängigkeit der auftretenden Singularitäten und beschrieb die Konfiguration der 27 Geraden und seine Entdeckung des sogenannten Doppelsechsers, was ihm später den Steiner-Preis eintrug.

Von den 27 Geraden können auf 72 Arten 6 paarweise windschiefe Geraden ausgezeichnet werden. $\mathrm{Zu}$ jedem solchen Sextupel $(1, \ldots, 6)$ gibt es ein Gegenstück $\left(1^{\prime}, \ldots, 6^{\prime}\right)$ windschiefer Geraden, welche zusammen einen der insgesamt 36 Doppelsechser bilden. Die 15 verbleibenden Geraden $g$ sind durch ihr Schnittverhalten mit den Geraden 
$1, \ldots, 6,1^{\prime}, \ldots, 6^{\prime}$ eines Doppelsechsers eindeutig bestimmt, nämlich als $g=(i j):=$ Gerade durch $i, i^{\prime}, j, j^{\prime}$ für $i<j$.

Dies kann am schönen Beispiel der Clebschen Fläche nachvollzogen werden. Die Fläche von Clebsch kann in homogenen Koordinaten dargestellt werden durch

$$
x_{0}^{3}+\ldots+x_{4}^{3}=0 \quad \text { mit der Nebenbedingung } x_{0}+\ldots+x_{4}=0 .
$$

Eine Gipsnachbildung der Clebschen Fläche aus der Rodenberger Serie ist in einem Schauschrank des Mathematischen Instituts in Göttingen zu bestaunen.

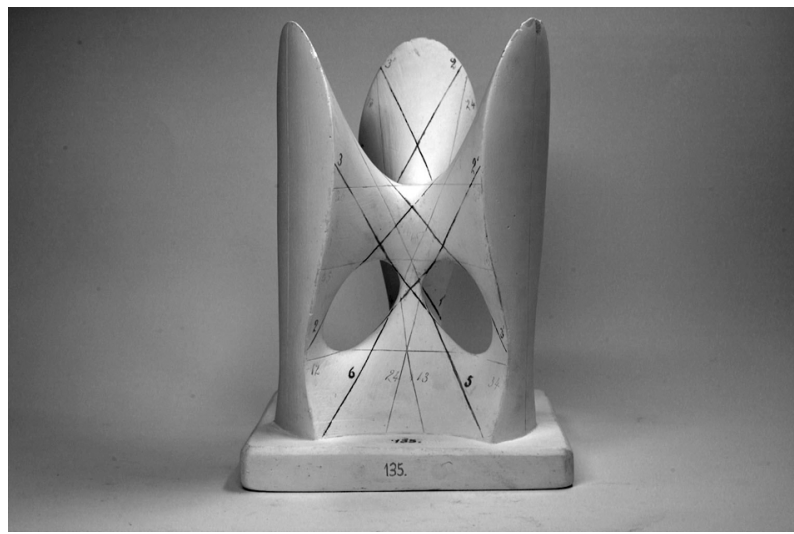

Abb. 2 Die Diagonalfläche von Clebsch. (Das Bild wurde vom Göttinger Mathematischen Institut zur Verfügung gestellt.)

Im Verlauf dieser Forschungen entdeckte Schläfli offenbar als erster die Nichtorientierbarkeit der projektiven Ebene, was er 1874 in einem Brief an F. Klein mitteilte.

II. Die Theorie der vielfachen Kontinuität tituliert Steiner begeistert die ,weltumstürmende, erdewälzende“. Sie ist in den Jahren 1850 bis 1852 entstanden. P.H. Schoute würdigt die Arbeit mit den Worten:

Diese Abhandlung übertrifft an wissenschaftlichem Wert einen guten Teil von allem, was bis heute auf diesem Gebiet der mehrdimensionalen Geometrie erschienen ist.

Schoute bedauert, dass die Arbeit nicht zu Lebzeiten Schläflis veröffentlicht werden konnte, gemäss:

So erfuhr denn dieser das tiefe Leid, das dem zufällt, der seiner Zeit voraus ist: Die Früchte seiner reifsten Studien nicht weltbekannt machen zu können. Und hierfür wird der Erfolg der Einteilung der kubischen Flächen nur eine kleine Entschädigung gewesen sein, weil diese, so verdienstvoll sie auch sein möge, nach meiner Meinung lange nicht so von Genie spricht, wie die Theorie der vielfachen Kontinuität. 
Tatsächlich wurde die „Theorie“ oder $\infty^{n}$, wie Schläfli sie gelegentlich auch bezeichnete, abgesehen von wenigen Auszügen vollständig erst 1901 veröffentlicht. Die 239 Seiten dicke Arbeit, die schwierig zu lesen ist und keinerlei Illustrationen enthält, wurde wegen ihres Umfangs weder von der Akademie in Wien noch von Crelle's Journal akzeptiert, was Schläfli sehr unglücklich machte.

Die „Theorie“ ist in drei Teile aufgeteilt und nach Schläflis Worten so,

wie wenn man etwa in der Geometrie 1. die Gerade und Ebene, 2. den Kreis und die Kugel, 3. die Kurven und Flächen zweiten Grades und endlich die infinitesimalen Eigenschaften der Kurven und Flächen überhaupt nacheinander behandeln würde.

Im ersten Teil entwickelt Schläfli die Geometrie im euklidischen Raum $\mathbb{E}^{n}$ der Dimension $n \geq 2$. Dazu gehören Untersuchungen über orthogonale Transformationen, die Definition des Abstands zwischen zwei Richtungen und Volumenformeln für Parallelotope und Pyramiden in Abhängigkeit der (Projektionen der) Kanten.

Zu den Hauptresultaten gehören zweifellos das $n$-dimensionale Analogon des Eulerschen Polyedersatzes und die Klassifikation und Konstruktion der regelmässigen Polytope im $\mathbb{E}^{n}$.

Der $\boldsymbol{n}$-dimensionale Eulersche Polyedersatz. Es sei $P \subset \mathbb{E}^{n}$ ein konvexes Polytop definiert als elementar-geometrische Summe von endlich vielen Simplizes. Es bezeichne

$$
\begin{aligned}
a_{i}=a_{i}(P) & :=\#\{i \text {-dimensionale Seiten von } P\}, \quad 0 \leq i \leq n-1, \\
a_{n} & :=1 .
\end{aligned}
$$

Dann gilt:

$$
a_{0}-a_{1}+\ldots+(-1)^{n} a_{n}=1
$$

Für $n=3$ ist dies der wohlbekannte Satz von Euler-Descartes, das heisst,

$$
a_{0}-a_{1}+a_{2}=2 .
$$

Die Klassifikation der regulären Polytope in $\mathbb{E}^{n}, \boldsymbol{n} \geq 2$. Induktiv ist der Begriff eines regulären Polytops wie folgt erklärbar:

Ein konvexes Polygon $P$ heisst regulär, falls alle Kanten und alle Winkel gleich sind. Ist die Kantenzahl gleich $p$, so schreibt Schläfli kurz $P=\{p\}$.

Ein $n$-dimensionales Polytop $P$ heisst regulär, falls alle Fazetten (Seiten der Dimension $n-1)$ regulär und alle Eckenfiguren regulär sind. Dabei entsteht die Eckenfigur zur Ecke $v \in P$, indem man gleiche Längen auf allen von $v$ ausgehenden Kanten abträgt und die konvexe Hülle der so entstehenden Endpunkte bildet.

Man beachte, dass alle Fazetten und alle Eckenfiguren eines regulären Polytops gleich sind. Schläfli führt anschliessend folgende Bezeichnung für reguläre Polytope $P_{\text {reg }} \subset \mathbb{E}^{n}$ ein, welche heute unter dem Begriff Schläfli-Symbol geläufig ist. 
Das Schläfli-Symbol eines regulären Polytops $P_{\text {reg }} \subset \mathbb{E}^{n}$ ist rekursiv definiert durch $P_{\text {reg }}=:\left\{p_{1}, \ldots, p_{n-1}\right\}$, falls

- die Fazetten gegeben sind durch $\left\{p_{1}, \ldots, p_{n-2}\right\}$, und

- die Eckenfiguren gegeben sind durch $\left\{p_{2}, \ldots, p_{n-1}\right\}$.

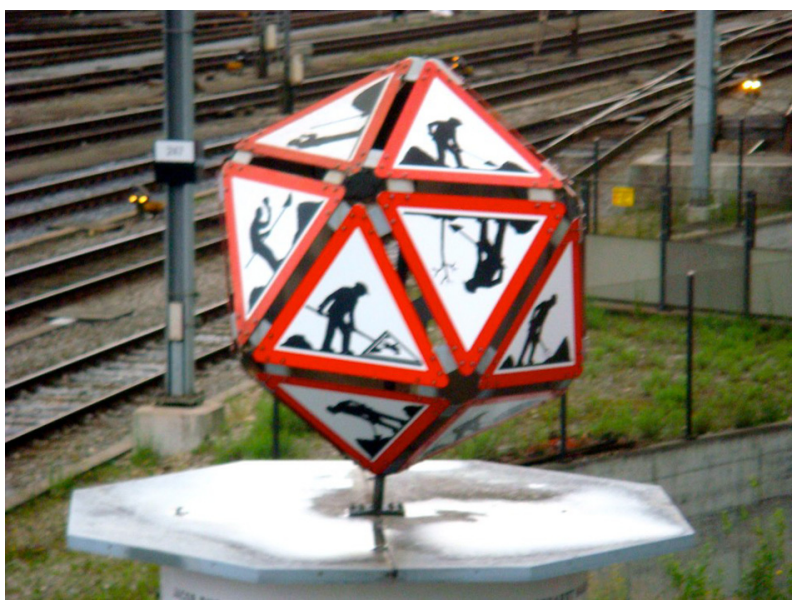

Abb. 3 Ein Ikosaeder $\{3,5\}$ beim Bahnhof SBB Basel, Juni 2010 (Photographiert von Elisabeth Kellerhals.)

Mit Hilfe der Eulerschen Polyederformel und mit kombinatorischen Relationen zwischen den Zahlen $a_{i}$ und $p_{j}$ für $P_{\text {reg }} \subset \mathbb{E}^{n}$, wie etwa $p_{2} a_{0}=2 a_{1}=p_{1} a_{2}$ für $n=3$, erhält Schläfli durch Induktion folgende uns heute wohlbekannte Tabelle:

\begin{tabular}{|c|c|c|}
\hline$n$ & Schläfli-Symbol & Bezeichnung \\
\hline 3 & $\{3,3\}$ & reguläres Tetraeder \\
& $\{4,3\}$ & regulärer Würfel \\
& $\{3,4\}$ & reguläres Oktaeder \\
& $\{5,3\}$ & reguläres Dodekaeder \\
& $\{3,5\}$ & reguläres Ikosaeder \\
4 & $\{3,4,3\}$ & $24-$ Zell \\
& $\{3,3,5\}$ & $600-$ Zell \\
& $\{5,3,3\}$ & $120-$ Zell \\
$\geq 5$ & $\{3, \ldots, 3\}$ & reguläre Pyramide \\
& $\{3, \ldots, 3,4\}$ & reguläres Kreuzpolytop \\
& $\{4,3, \ldots, 3\}$ & regulärer Hyperwürfel \\
\hline
\end{tabular}

Weiter findet Schläfli vier der insgesamt zehn regelmässigen Sternpolytope, die schon von Kepler und Poinsot entdeckt und von Hess schliesslich vollständig klassifiziert worden sind (cf. [2]). 
Im zweiten Teil beschäftigt sich Schläfli mit $n$-dimensionaler sphärischer Geometrie. Er führt zunächst sphärische Koordinaten ein und betrachtet dann bald das Volumenfunktional auf der Menge der Simplizes auf der Sphäre $\mathbb{S}^{n}$.

Es sei $S \subset \mathbb{S}^{n} \subset \mathbb{E}^{n+1}$ ein Simplex berandet von $n+1$ Hyperebenen $H_{i} \cap \mathbb{S}^{n}, H_{i} \subset \mathbb{E}^{n+1}$ mit $H_{i}=e_{i}^{\perp}$, und mit Diederwinkeln $\alpha_{i j}=\angle\left(H_{i}, H_{j}\right)$. Die Diederwinkel von $S$ treten an den Seiten $F_{i j}=H_{i} \cap H_{j} \cap S$ auf.

Schläfli beweist nun folgenden fundamentalen Satz:

\section{Die Differentialformel von Schläfli.}

$$
d \operatorname{vol}_{n}(S)=\frac{1}{n-1} \sum_{i<j} \operatorname{vol}_{n-2}\left(F_{i j}\right) d \alpha_{i j}, \quad \operatorname{vol}_{0}(\{\text { Punkt }\}):=1 .
$$

Insbesondere für sphärische Dreiecke $\Delta=\Delta(\alpha, \beta, \gamma)$ ergibt sich

$$
d \operatorname{vol}_{2}(\Delta)=d \alpha+d \beta+d \gamma,
$$

woraus man unschwer die bekannte Exzessformel

$$
\operatorname{vol}_{2}(\Delta)=\alpha+\beta+\gamma-\pi
$$

ableiten kann. In einem Brief an Steiner schreibt Schläfli über seine Entdeckung Folgendes:

Ich glaube, den erwähnten allgemeinen Satz nicht zu überschätzen, wenn ich ihn dem Schönsten, was in der Geometrie geleistet worden ist, an die Seite stelle.

Für diesen Satz gibt Schläfli zwei Beweise an, einen differentialgeometrischen und - 15 Jahre später - einen funktionentheoretischen. Der zweite Beweis befindet sich in einer Arbeit über Besselfunktionen, Über die Entwickelbarkeit des Quotienten zweier bestimmter Integrale von der Form $\int d x d y \ldots d z, 1867$, im dritten Band der Abhandlungen.

Die Differentialformel vereinfacht sich, wenn man die normierte Schläflische Funktion für sphärische $n$-Simplizes $S$

$$
f_{n}=f_{n}(S):=\frac{2^{n+1}}{\operatorname{vol}_{n}\left(\mathbb{S}^{n}\right)} \operatorname{vol}_{n}(S)
$$

benützt, welche

$$
f_{n}=1 \quad \text { für } \text { orthogonale Simplizes } \quad\left(\text { alle } \quad \alpha_{i j}=\frac{\pi}{2}\right)
$$

erfüllt. Damit folgt

$$
d f_{n}=\sum_{\sigma} f_{n-2}(\sigma) d f_{1}\left(\alpha_{\sigma}\right),
$$

wobei die Summe über alle $(n-2)$-dimensionalen Seiten von $S$ erstreckt wird, und $\alpha_{\sigma}$ den Diederwinkel an der Seite $\sigma$ von $S$ bezeichnet. 
Für die sphärische Simplexfunktion $f_{n}$ beweist er im weiteren einige schöne Eigenschaften. Sind beispielsweise die Normalenvektoren $e_{i}$ zu den berandenden Hyperebenen $H_{i}$ von $S$ so beschaffen, dass

$$
e_{0}, \ldots, e_{m} \perp e_{m+1}, \ldots, e_{n}, \quad 0 \leq m \leq n-1,
$$

so gilt für $f(0 \cdots n):=f_{n}(S)$ die Produktregel

$$
f(01 \cdots m) f([m+1] \cdots n)=f(0 \cdots n) .
$$

Mit diesen Eigenschaften und der Differentialformel gelangt Schläfli schliesslich zum zweiten Hauptsatz:

Die Reduktionsformel von Schläfli. Ist $S$ ein $(2 n)$-dimensionales sphärisches Simplex, so gilt

$$
f_{2 n}(S)=\sum_{k=0}^{n}(-1)^{k} a_{k} \sum_{V} f_{2(n+k)-1}(V), \quad \sum f_{-1}:=1,
$$

wobei $V$ alle (sphärischen) Eckenfiguren der ungeraden Dimensionen $<2 n$ durchläuft, und wobei die $a_{k}$ gegeben sind durch Tangentenzahlen vermöge

$$
\tan x=\sum_{k=0}^{\infty}(-1)^{k} a_{k} \frac{x^{2 k+1}}{(2 k+1) !} .
$$

Die Koeffizienten $a_{k}$ sind ganzzahlig und mit Bernoulli-Zahlen verwandt gemäss

$$
a_{k}=2^{2 k+1} \cdot \frac{2^{2 k+2}-1}{k+1} \cdot B_{k+1} 1 \quad(=1,2,16,17 \cdot 16, \ldots)
$$

Für ein sphärisches Dreieck $\Delta=\Delta(\alpha, \beta, \gamma)$ folgt aus der Reduktionsformel, dass

$$
f_{2}(\Delta)=f_{1}(\alpha)+f_{1}(\beta)+f_{1}(\gamma)-2 \text { und somit } \operatorname{vol}_{2}(\Delta)=\alpha+\beta+\gamma-\pi .
$$

Es ist allerdings ein Rätsel, wie Schläfli auf die explizite Form der Koeffizienten $a_{k}$ gestossen ist. Dahinter stecken viele Berechnungen, die im endgültigen und sehr eleganten Induktionsbeweis unterdrückt werden können. In der Tat reicht im Nachhinein dank der Differentialformel eine gewisse Rekursionsformel für die Koeffizienten $a_{k}$ aus, um diese mit Tangentenzahlen zu identifizieren.

Mit Hilfe der Differential- und der Reduktionsformel ist Schläfli imstande, Volumenrelationen für seine Orthoscheme herzuleiten. Orthoscheme sind Simplizes beschrieben durch $n+1$ Einheitsnormalenvektoren $e_{i} \in \mathbb{E}^{n+1}$ mit der Eigenschaft

$$
e_{i} \perp e_{j} \quad \text { für } \quad|i-j|>1 .
$$

Für die Diederwinkel folgt daraus, dass $\alpha_{i j}=\frac{\pi}{2}$ für $|i-j|>1$. Orthoscheme sind somit gegeben durch höchstens $n$ nicht-rechte Diederwinkel $a_{i}:=\angle\left(H_{i-1}, H_{i}\right)$ für $1 \leq i \leq n$. Sie bilden einen Polyederbaukasten.

In der heutigen modernen Sprache ausgedrückt ist er schliesslich imstande, alle Kovolumina (oder Ordnungen) der endlichen Coxetergruppen mit linearem Coxeter-Schläfli- 
Diagramm

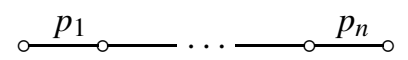

zu berechnen. Zum Beispiel erhält er, für $n \geq 1$,

$$
\begin{aligned}
f_{n}\left(\frac{\pi}{3}, \ldots, \frac{\pi}{3}\right) & =\frac{2^{n+1}}{(n+2) !}, \\
f_{n}\left(\frac{\pi}{4}, \frac{\pi}{3}, \ldots, \frac{\pi}{3}\right) & =\frac{1}{(n+1) !} .
\end{aligned}
$$

Schläfli vermutet, dass dies für $n \geq 4$ die einzigen Formeln sind, worin sowohl die Argumente kommensurabel mit $\pi$, als auch die $f_{n}$-Werte rationale Zahlen sind. Diese Vermutung, die heute die Rational Simplex Conjecture genannt wird, ist immer noch offen und - wie Schläfli selbst schon angedeutet hat [8, Vol. I, S. 267] - sehr schwierig zu beweisen! Im dritten und letzten Teil behandelt Schläfli verschiedene Anwendungen seiner Theorie auf quadratische Kontinua. Er beweist Aussagen über die Hauptachsen, Mittelpunkte und konjugierten Durchmesser solcher Gebilde und verallgemeinert einige bekannte Resultate von Monge, Chasles und Dupin von drei auf höhere Dimensionen. Neben Sylvester und Gauss kann er auch als Mitentdecker des Trägheitsgesetzes für quadratische Formen angesehen werden, für welches er einen Beweis mit Hilfe von Stetigkeitsbetrachtungen angibt. Darauf möchte ich jetzt aber nicht weiter eingehen. Vielmehr möchte ich zum Schluss Schläflis zweite Lebenshälfte nacherzählen.

\section{Die zweite Lebenshälfte}

Schläflis Lebensumstände verbessern sich langsam. Nach seiner Berufung zum ausserordentlichen Professor im Jahre 1853 wird er mit 54 Jahren zum Ordinarius befördert, und ab 1879 erhält er endlich ein einigermassen standesgemässes Gehalt. Als Universitätslehrer setzt er sich sehr ein und ist überaus beliebt. Er unterrichtet durchschnittlich 10 Stunden pro Woche, und dies auf sehr lebendige Weise. Sein Schüler Graf berichtet dazu Folgendes:

Er bewegte sich mit jugendlicher Behendigkeit an der Wandtafel, und da er von kleiner Statur war, mußte er sich oft des Stuhles bedienen, um mächtige Ausdrücke von Formeln auf der Tafel unterzubringen. Er war ein fabelhafter Kopfrechner, und nach seinem eigenen Geständnis, arbeitete er jedes Kolleg (Vorlesung), wenn er es zum zweiten oder dritten Male hielt, neu um.

Schläfli selbst macht dazu folgende Bemerkungen:

\section{Schläfli an C.W. Borchardt}

Was die rein wissenschaftliche Tätigkeit betrifft, so steht ihr die allgemeine Ermüdung des Geistes, daher die Häufigkeit logischer Irrtümer und Schwerfälligkeit des Herauswindens aus wirklichen oder vermeintlichen Widersprüchen entgegen. Ich bin über pflichtmässige Unterrichtstätigkeit äusserst froh und bemühe mich, meine mathematische Hartknochigkeit und Routine auf andere zu übertragen. Ich trachte immer im Unterricht so frisch und unmittelbar als möglich zu sein und, wenn es mir gelingt, mich in dieser Tätigkeit aufzubrauchen, so will ich mich für glücklich halten. 
Schläfli hatte 12 Doktoranden, davon wurden 6 Universitätsprofessoren - eine für zumindest damalige Verhältnisse erstaunlich grosse Zahl. Steiner dagegen hatte keine eigenen, direkten Doktoranden. Neben Graf möchte ich Schläflis Doktoranden Arnold Meyer nennen. Seine Dissertation über indefinite ternäre Formen wurde später für die Arbeiten von Martin Eichler sehr bedeutungsvoll.

Schläfli erfährt nun auch zunehmend und zahlreich Ehrungen aus dem In- und Ausland. Im Jahre 1863 wird ihm die Ehrendoktorwürde der philosophischen Fakultät Bern verliehen.

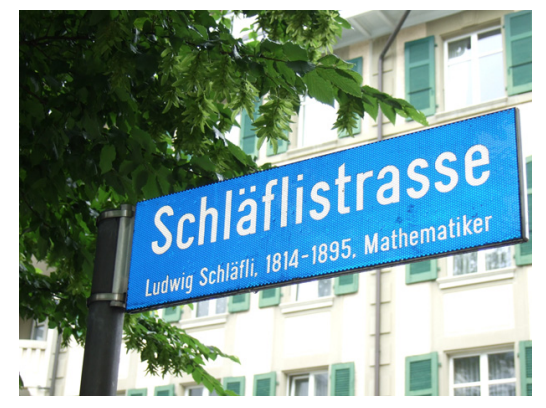

Abb. 4 Die Schläflistrasse in Bern, Juni 2010

Schläfli wird ernannt zum Korrespondierenden Mitglied des Reale Istituto Lombardo di Scienze e Lettere, Mailand (1868), der Königlichen Gesellschaft der Wissenschaften zu Göttingen (1871) und der Reale Academia dei Lincei, Rom (1883). Ebenso im Jahr 1883 erhält er von der Berliner Akademie der Wissenschaften den Steiner-Preis von 600 Thalern für seine Arbeiten über die Kubiken. Und im Jahr 1889 wird er Ehrenmitglied des Komitees bei der 100-jährigen Gedenkfeier von N.I. Lobatschewskij, einem der Begründer der hyperbolischen Geometrie.

Gesundheitlich geht es Schläfli im höheren Alter immer schlechter. 1891 lässt er sich deshalb pensionieren. Er leidet an Atemnot und Wassersucht, und eine alte Beinbruchverletzung, die er gedankenverloren beim Zusammenstoss mit einem Betrunkenen erlitten hat, fesselt ihn ans Bett. Bis zuletzt ist er geistig hellwach. Er stirbt am 20. März 1895.

Ich möchte diesen kurzen Beitrag schliessen mit folgendem Auszug aus Grafs Gedenkrede [4] bei der Denkmalseinweihung an Schläflis Grab am 18. März 1896.

\section{Auszug aus Grafs Rede}

Und was war er als Mensch! So unscheinbar und bescheiden im Auftreten, eine wahre Gelehrtennatur, nur glücklich in der Stille des Studierzimmers. Wer sein Schüler sein durfte, weiss, welche Aufopferung und Hingebung, gepaart mit Herzensgüte, in diesem Manne wohnten. Sein gerader und offener Charakter verabscheute jede Ungerechtigkeit, am Vaterlande hing er treu, ihm wollte er dienen, darum allein schlug er die ehrenvolle Berufung in fremde Lande aus. Über sein Leben lässt sich das Motto setzen, das in der Gratulationsschrift zu seinem 70. Geburtstage in Sanskrit geschrieben steht: Die Wissenschaft ist des Mannes schönste Schönheit, ein tief geborgener Schatz. Man darf wohl sagen, seit des unvergleichlichen Leonhard Euler's Tode hat kein Schweizer wie er das mathematische 


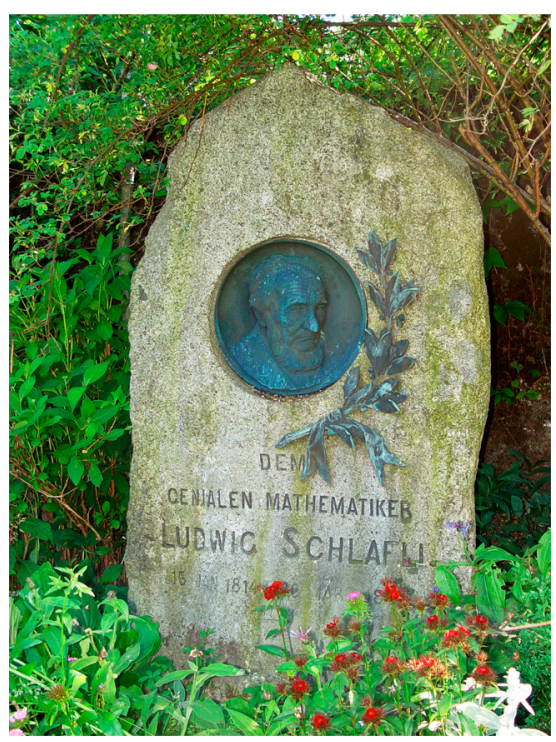

Abb. 5 Dem genialen Mathematiker Ludwig Schläfli, Inschrift auf dem Grabstein in Heimiswil, Juni 2010. (Photographiert von Norbert Hungerbühler im Park des Landgasthofs zum Löwen in Heimiswil (Kanton Bern).)

Wissen beherrscht und unter den zeitgenössischen Mathematikern kann man ihm nur Wenige, was die Mannigfaltigkeit der durchforschten Gebiete anbetrifft, an die Seite stellen.

\section{Literatur}

[1] Burckhardt, J.J.: Ludwig Schläfli. Beiheft 4 zu Elem. Math., Basel 1948.

[2] Coxeter, H.S.M.: Star polytopes and the Schläfli function $f(\alpha, \beta, \gamma)$. Elem. Math. 44 (1989), $25-56$.

[3] Graf, J.H.: Ludwig Schläfli. Mitteilungen der Naturforschenden Gesellschaft in Bern (1896), 118-203.

[4] Graf, J.H.: Die Exhumirung Jakob Steiner's und die Einweihung des Grabdenkmals Ludwig Schläfli's. Mitteilungen der Naturforschenden Gesellschaft in Bern (1897), 6-24.

[5] Kellerhals, R.: Der Mathematiker Ludwig Schläfli (15.01.1814-20.03.1895). DMV-Mitteilungen 4 (1996), 35-43.

[6] Rytz, W.: Prof. Ludwig Schläfli als Botaniker. Mitteilungen der Naturforschenden Gesellschaft in Bern (1919), 213-220.

[7] Schlaginhaufen, O.: Der Schädel des Mathematikers Ludwig Schläfli. Mitteilungen der Naturforschenden Gesellschaft in Bern (1931), 35-66.

[8] Schläfli, L.: Gesammelte Mathematische Abhandlungen. 3 Bände, Birkhäuser, Basel 1950-1956.

Ruth Kellerhals

Departement für Mathematik

Universität Freiburg

Museumsweg 23

CH-1700 Freiburg, Schweiz

e-mail: ruth.kellerhals@unifr.ch 